\section{METHODS OF MEASURING ATMOSPHERIC POLLUTION BY SUSPENDED MATTER. ${ }^{1}$}

BY Dr. John S. OwENs, F.R.G.S., F.G.S., A.M.I.C.E., M.R.S.I.

IN this paper some of the different methods which may be used to measure the degree of pollution of the air by smoke, dust, and other suspended impurities are considered, and their relative merits discussed.

In 1911, at the International Conference on Smoke Abatement held in London, it was proposed by the author that an attempt should be made to obtain by some uniform and standardised method comparative data as to the degree of pollution of the air at different parts of the United Kingdom. A committee was appointed at the conference to consider what means should be adopted to attain this object, and the author undertook the work of honorary secretary. In connexion with this work it became necessary to consider methods of measurement and to decide on a standard form of apparatus to be adopted. The results of the investigations made appear in this paper.

Before reviewing the possible methods which might be used it will be well to define exactly the objects in view. These were: (a) To measure quantitatively the amount of matter deposited from the air at any place during a fixed period; (b) to measure the amount of matter held in suspension in the air at any time and place; and (c) to find the nature and composition of deposited or suspended matter.

A knowledge of the degree of pollution of the air at different places enables us to make a comparison between one place and another, and also gives some measure of the success attending attempts to purify the air in any particular district.

Having defined the objects in view we may now discuss the various methods and their relative merits in the light of this definition.

1. A measured volume of air may be filtered through cotton or asbestos wool and the weight of the deposit ascertained. This method was used by Mr. Russell for the Meteorological Office, and the details of his experiments are given in the monthly weather reports for 1884-85; it has also been used since by different observers. In order to ascertain the weight of deposit on the filter the wool has to be very carefully dried and weighed before and after the air is drawn through, and it is in this part of the process that the greatest difficulties arise. It is by no means easy to get the wool dried completely, and the amount of matter caught from the air by the filter is so small that large errors may be thus introduced. There is also the difficulty of obtaining a filter which removes all the suspended matter from the air. In experiments made by the author in conjunction with Mr. J. C. Olark in 1910 the filtering medium used. was a cotton wool plug in a glass tube. In order to dry this thoroughly air was drawn through a tower containing $\mathrm{CaCl}_{2}$, then through $\mathrm{H}_{2} \mathrm{SO}_{4}$, and then through the cotton wool filter, which was kept warmed in a special water bath. To test the efficiency of the filter, air was drawn through two filters placed in tandem; in case the first was not stopping all the suspended matter, the second filter should show an increase in weight after a considerable rolume of air had been drawn through. The air was drawn through the apparatus by means of a reversed gas compressor driven by a small hot-air engine, and between the pump and the filter a gas meter was fix sd to measure the volume dealt with. The method worked fairly well, but it was soon found that to obtain reliable results a larger apparatus would be required, one capable of filtering at least 100 cubic feet per hour. In one observation made in London on Dec. 28th, 1910 , during a slight fog, $26 \cdot 1$ cubic feet of air were filtered and an increase of weight of only 0.0014 gramme obtained. By this method, although the amount of impurity can be ascertained, it is not easy to find its nature owing to the difficulty in separating it from the filter. It may be said to fulfil condition $(b)$ but not $(a)$ or $(c)$. In fact, no single method can fulfil both conditions $(a)$ and $(b)$; that is,

1 A paper read at the meeting of the British Association for the Adrancement of Science. measure the amount of deposited and suspended impurity. Two separate observations are required, but any method used for either (a) or (b) should also fulfil conditions ( $b$ ), as it is important to find the nature of the impurity present.

The Method adopted as the Standard.

2. All rain and deposited matter falling on a gauge vessel of known catchment area may be collected, the water evaporated or filtered, and the residue weighed and analysed. An investigation was conducted by THE LANCET during the years 1910 and 1911 into the sootfall of London, this method being used. The experiments were made under the auspices of the Coal Smoke Abatement Society, and all the analytical work was done in THE LANCE' Laboratories under the direction of Mr. Vasey. The gauge was designed by the author, and consisted of a vessel of 4 square feet catch. ment area, having a conical bottom so arranged that al water falling in the gauge vessel was collected in bottles placed underneath. These bottles were removed at intervals of one month and the contents analysed. The gauge vessel was of enamelled iron, square in plan, and supported in a frame on four iron legs; it was surrounded by a cage of wire netting, open at the top to prevent birds from con. taminating the deposit.

It was at first thought that the gauge might be fitted with a filter which would retain all the solid matter washed into it by the rain, allowing the water to escape. For this purpose a glass vessel $3 \mathrm{in}$. in diameter and $6 \mathrm{in}$. deep, terminating in a conical bottom with a small central opening, was fixed to the bottom of each gauge; this vessel was fitied with a filter of fine glass wool held between two screens formed of nickel gauze with asbestos wool between. It was pointed out, however, by Mr. S. A. Vasey, the director of 'THE LANCET Laboratories, that this filter frequently showed a loss instead of a gain in weight after receiving a month's rainfall. This, he suggested, was probably due to solubility of the glass wool. The method was therefore abandoned in favour of that ultimately suggested by $\mathrm{Mr}$. Vasey, in which all water and deposit were retained for examination. As modified in this way, it has now been adopted by the Committee for the Investigation of Atmo. spheric Pollution, and with certain other alterations in the form and details of construction, as indicated below, has been decided on as the standard method for use by authorities which are coöperating with the committee by taking observations in their respective districts. It fulfils conditions $(a)$ and $(a)$.

The standard form of apparatus decided on consists of a circular gauge vessel of enamelled cast iron, the enamel being an insoluble porcelain. The gauge vessel rests in a circular ring supported on four legs, which also carry a shelf for holding the bottles which collect the water and deposit A cage of wires surrounds the top of the apparatus to prevent birds from settling on the edge of the gauge. This is illustrated in Fig. 1. Simultaneous observations will be commenced at from 20 to 30 different stations in the United Kingdom on Oct. 1st next, and it is hoped that very valuable results will be obtained. The gauge collects rain water and any impurities carried down by it, and dust or soot which fall in the vessel during dry weather. No attempt is to be made at present to distinguish between these two deposits, but it is not unlikely that when the work is well established the committee will consider the possibility of separating them.

When removing the bottles at the end of each month the gauge vessel will be washed down carefully into the bottles by using some of the water already collected, so as to remove any adherent matter. The results obtained during the pre liminary year's experiment above referred to, made with gauges of this type, were very promising. The monthly deposits at four different stations were compared; tbree of these stations were in London, the fourth in my garden at Cheam, Surrey, about 12 miles out on the S.W. side. The total amounts of deposit for the year, according to the estimations made in THE LANCET Laboratories, were as follows:-

Station.

Deposits in tons per square mile.

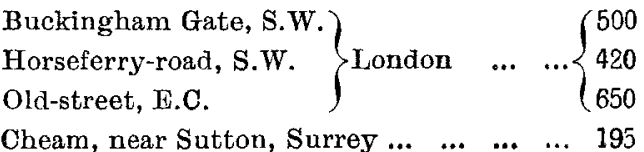

2 See The Lancet, Jan. 6th, 1912. 
The curves (Fig. 2) show the sootfall and rainfall for each month at the three London stations (averaged) ano at Cheam, Surrey. It will be seen that the deposit on the London area is very much greater than at Cheam. Also its composition is different, as the deposit in London contained about six times as much soot, sulphate, and ammonia. There is also an indication of a relation between the amount of rain and of solid matter deposited, but the period is not long enough to enable us to state what the relation is. It is hoped that when the results from different centres and for longer periods are available the exact nature of this relation will be brought out.

\section{Other Methods.}

3. Aitken's dust counter.-This is an ingenious instrument devised by Mr. John Aitken and described fully in the Transactions of the Royal Society of Edinburgh, Vols. XXX. to XXXVI. It is intended for counting the number of dust particles in the air, but no attempt is made to ascertain their composition. It is questionable whether the number of particles is a fair measure of the amount of impurity present, since no account is taken of their size. Hence it would appear that a small number of large dust particles may, according to this instrument, indicate a purer air than if a large number of smaller particles were present, whereas the amount of impurity may be greater in the former than in the latter case. According to Aitken there are about 300 to 3000 dust particles in a cubic centimetre of country air from Argyleshire, whereas that of London contains 48,000 to 150,000 in the same volume of air. The method adopted is to cause the moisture in a small measured volume of air to be precipitated as rain on the glass slide of a small microscope, when the number of drops which fall may be counted. It is assumed that each drop has for its nucleus a dust particle. The method has the drawbacks above indicated, and cannot be said, from our present point of view, to fulfil any of the conditions laid down.

4. Glass plates may be exposed to the air for a known length of time, then washed in water and their opacity measured. This method was devised by Professor Cohen, of Leeds, and used there by him. It is primarily intended for measuring the amount of tarry soot which falls at a given place; as tar, or some sticky matter, is necessary to enable the deposit to adhere to the plate when washed. Obviously the results cannot be based on the opacity of the unwashed plate, since it must be always exposed to rain, and will therefore be more or less washed whether it is intended to be or not. The indication given provides a rough basis of comparison between the deposits at different places; also since the chief distinguishing feature between smoke or soot from a domestic chimney and that from boiler and other industrial furnaces is the percentage of tar present, the method enables us to say roughly whether the deposit is due chiefly to domestic or factory smoke. There are, however, certain objections which make it unsuitable as a standard method. Briefly, none of the conditions laid down are fulfilled; the opacity of the plate must depend on the stickiness of the deposit, not on its amount; also it appears probable that during the period when the greatest amount of deposit reaches the ground-that is, during rain-the plate would be unable to retain it owing to its surface being wet.

5. A jet of air of standard size and velocity may be caused to strike a glass plate placed at a fixed distance from the nozzle, and the opacity of the plate measured after a definite time. The plate might be coated with some sticky substance, so that all particles striking it adhere. This method might be used in one of two ways: The air jet may be caused to play on the plate for a fixed time and the opacity measured by comparison with a specially calibrated scale, or the jet may be made to play on the plate for such a time as will cause a fixed opacity, and the time required compared with a calibrated time scale. So far as I am aware the method has not been tried, and is now suggested for discussion. Some such means of obtaining the amount of suspended matter, as distinct from that deposited, is required ; and it should be one which permits of making a complete measurement in a short time, such as an hour, since in cities where fogs are prevalent it is usual to find great variations in their density occurring during short periods. Practically speaking, the shorter the time required for making an observation the more suitable the method would be ; it is probable, however, that the one described above would not give sufficient accuracy to be suitable.

6. A measured volume of air may be drawn through filter paper and the degree of discolouration produced on the paper compared with a calibrated scale. Or the air may be drawn through the paper until a predetermined degree of discolouration has been produced. The method has been tried in Glasgow with considerable success, and experiments are now being carried out with a view to its adoption as a standard

FIG. 1.

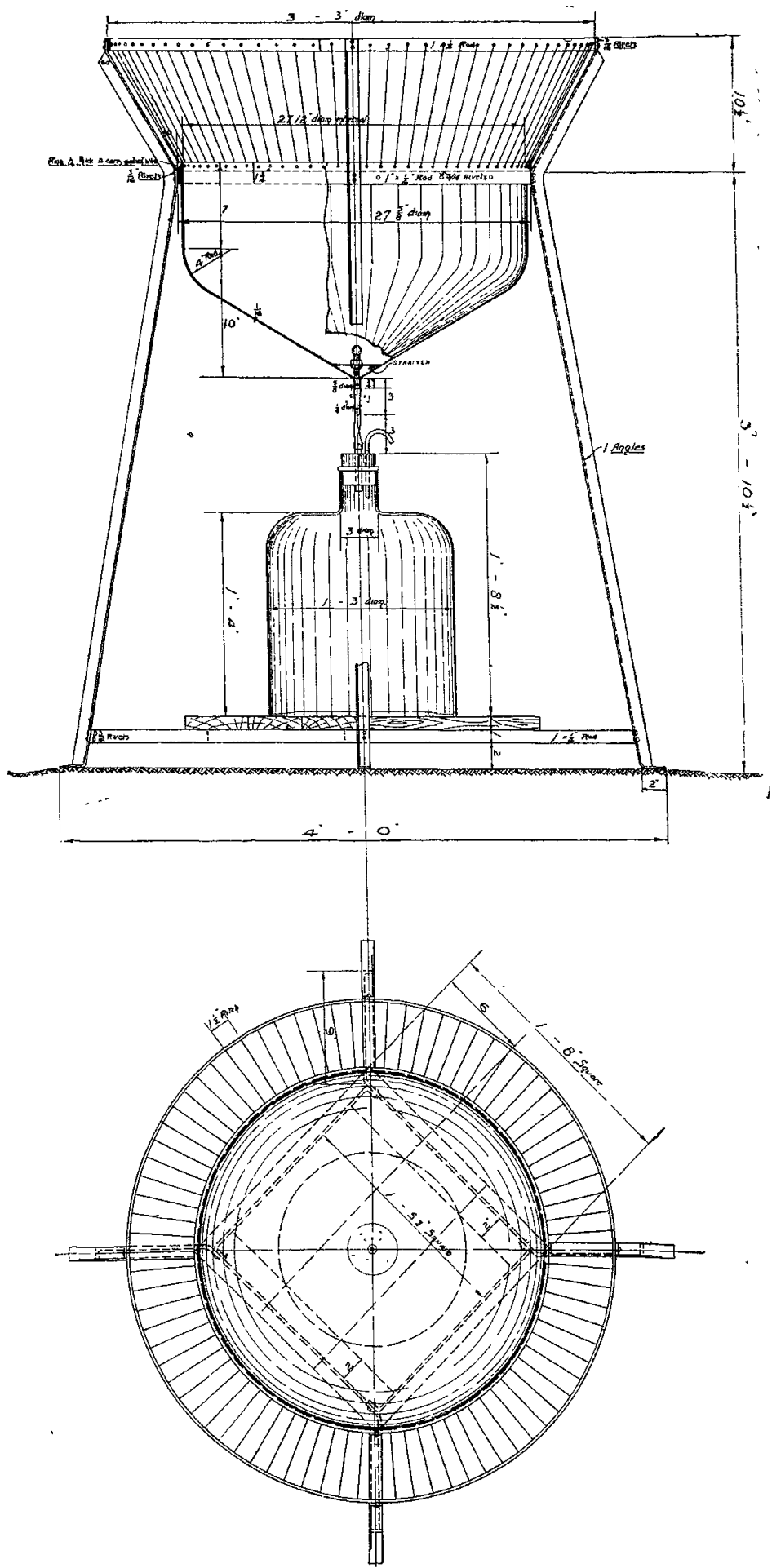

Standard deposit gauge as adopted by the Committee for the Measurement of Atmospheric Pollution.

by the Committee for the Investigation of Atmospheric Pollution. There are, however, certain difficulties and drawbacks which have yet to be overcome. In the experiments made the air was drawn through a sort of drum over the mouth of which discs of filter paper could be fixed. The air was measured by passing through a meter, the pump being operated by a small electromotor. After some time the paper becomes quite black and its pores somewhat clogged up, so that it is difficult to maintain a current of uniform velocity through it. Also the colour of the deposit depends on other factors besides its amount; in fact, it is only when the nature of the suspended matter is the same that a comparison may be made between different places by this means. It is, however, fairly suitable for comparing the degree of pollution 
of the air at the same place at different times. The attempt $/$ method, the chief advantage being that, if practicable is now being made to obtain a suitable filter paper which it would permit an observation to be taken almust may be dissolved in some liquid, and by this means separated instantaneously, and would require no weighing to give the from the deposit; but this is difficult, since it must be some- degree of pollution; it would not, however, fulfil condition thing which will not interfere with the subsequent analysis $(c)$, which is most important. Also, the indication given of the deposit. The amount of deposit on a filter paper would depend rather on the power of the suspended matter which is required to cause a definite discolouration is so to obstruct light than on the quantity present; in other small as to be hardly weighable. With some modification words, the size of the suspended particles would falsify the such as above indicated, and provision for ubtaining a suffi- result, since a small number of large particles might contain cient quantity of deposit to weigh with accuracy, this method more impurity than a large number of smaller ones, while promises to be very useful, as the time required for an obstructing less light. That this is no fanciful objection

Fic. 2.-Curves showing Suotfall and Rainfall for each Month at three London Stations, and at Cheam, Surrey, accordiny to Analyses made in The LANCET Laboratory.

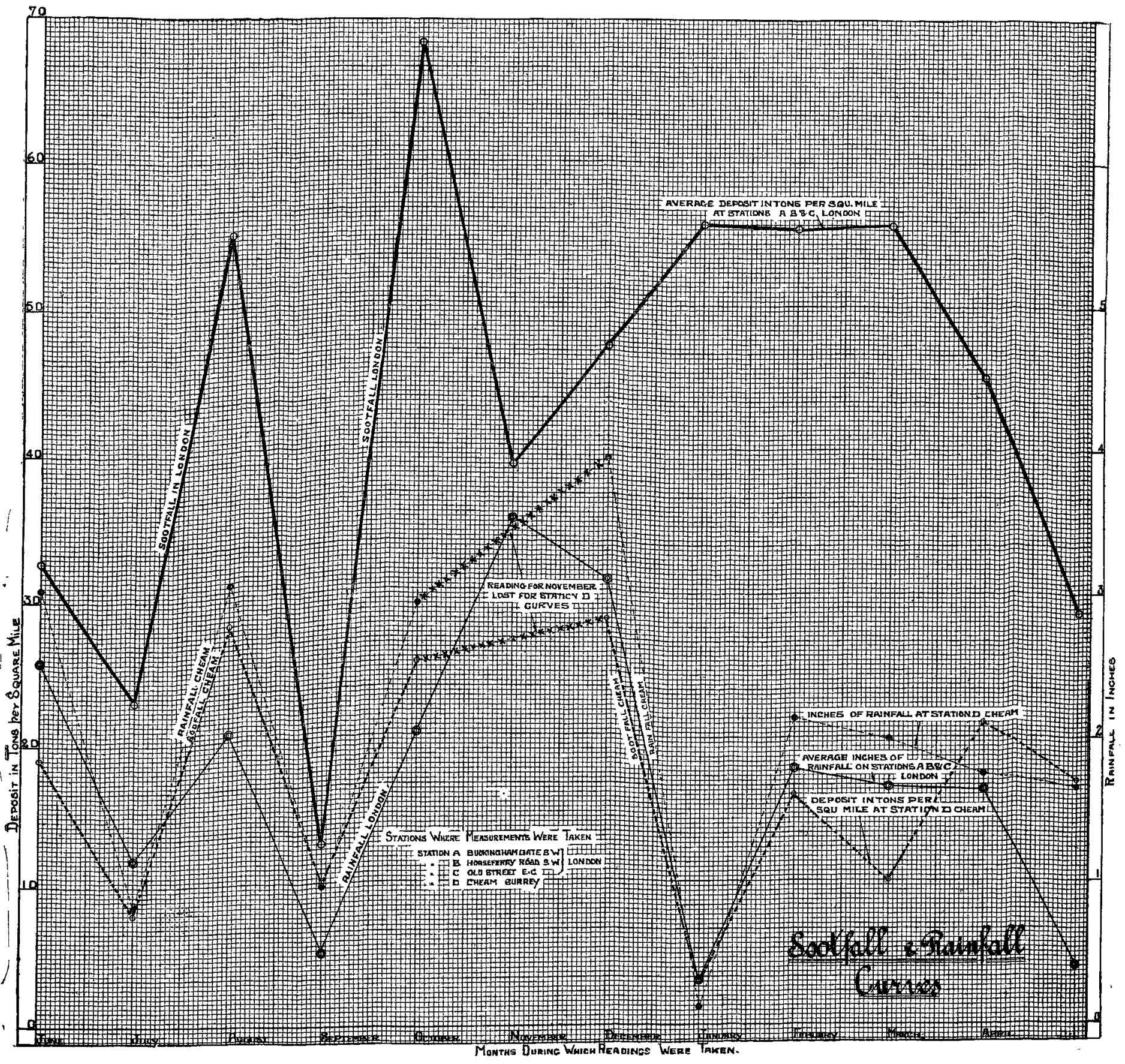

observation is short, and the whole apparatus may be made in"a compact form.

7. An optical method might be used by which the opacity of a column of air of a given length to a standard light is measured. This might be arranged to give quantitative results by preparing a scale of opacity from measurements taken on air with known amounts of suspended matter present, but the results would probably not be of great accuracy, unless perhaps in the case of dense smoke fogs. The presence of water vapour would have to be guarded against in some way, out how it is not easy to see. It might perhaps be done oy keeping the column of air warmed so as to destroy the part of the fog due to water, and agitated to prevent settling of the other particles while making an observation. It is not a very promising may be seen by anyone who notes the variation in the size of smuts which fall in cities on different days. It seems probable that under certain atmospheric conditions soot, and probably other kinds of suspended matter, tend to collect in large particles, whereas on other days there is no such tendency. It would seem from this consideration that no method of measuring suspended matter which depends on the opacity of the air, or of glass plates exposed to catch the deposit, or on the degree of discolouration of filter paper through which air is drawn, can be of great accuracy; and the indication is that whatever method is used it must aim at the weighing and analysis of the matter suspended in a known volume of air.

8. The rain might be caught and its opacity, afte thorough shaking, compared with a standard scale made by 
adding definite quantities of soot to distilled water. A method somewhat similar to this was used by Dr. Fritzsch for measuring smoke issuing from chimneys. He filtered a measured volume of smoke through filters of cellulose, which was subsequently dissolved in ether and the opacity compared with other solutions containing weighed quantities of soot. For our present purpose the method is open to objection, as it only gives the quantity of impurity carried down, and that with doubtful accuracy, while it does not fulfil condition $(c)$. The apparatus required would also be almost similar to that described under method 2 , but the results would not be so valuable.

9. Boxes having a collecting surface of known area may be exposed for a definite time and their contents collected and analysed. This system was devised by Mr. Peter Fyfe, chief sanitary officer, Glasgow, and was used with some success in that city. It is a crude form of method 2. The boxes to be effective must be watertight, otherwise during dry, windy weather some of the collected material would be blown away. In this form they are simply rain and deposit gauges aiming at the same results as method 2 , except that in the present case it is necessary to remove the whole gauge-box when it is desired to measure the amount of deposit, whereas in the other the rain and deposit are allowed to fall into bottles placed under the gauge vessel, and these are removed for analysis.

\section{Remarks.}

In conclusion, there are one or two points which it will be well to keep in mind when considering the subject of this paper. The utmost care is now lavished by our public health authorities in preventing pollution of our drinking water and of food; but while we consume these to only a moderate extent as compared with the air which we breathe, very little attention is given to purifying or preventing pollution of the latter. Curiously enough also the whole science of ventilation, if it may be called a science, is based upon an utter fallacy. We now know, without any question of doubt, that the "terrible" gas-carbon dioxide-which is still used as an indicator of good or bad ventilation is quite harmless unless when present in a proportion approaching that in expired air. It is unnecessary to dwell on this point, since it is beyond question. We also know that all air-borne diseases are transmitted, not by means of a poisonous gas, but by solid particles carried in suspension in the air. Yet still we find ventilation experts gravely measuring the proportion of $\mathrm{CO}_{2}$ in the air of a building, and pronouncing from this on the suitability or otherwise of the air tor breathing. Surely it is time for us to alter all this, and base our statements as to the purity of the air upon the absence of dust, which is the real source of danger.

One other point may be mentioned. When a person is exposed to infection - for example, breathes an air laden with tubercle bacilli-whether he contracts the disease or not depends on two factors: (a) the dose, and perhaps the virulence of the disease carrying microbe, which he receives and $(b)$ his own resisting power to infection.

It is important to remember these, as too much stress is often laid on one and not enough on the other. There are now several systems of ventilation by which an attempt is made to filter the air before it is allowed to enter a building but no attempt is made to measure what the purifying effect of such methods is. The designer appears to be satisfied if his screens or filters collect a good deposit of dirt. As it is impossible by ordinary means to distinguish between dust which is more or less harmless and that which contains disease germs, surely it is not going too far to say that careful measurements of the amount of suspended impurity contained in the air would be a far better indication of its suitability for breathing than our present methods. Such measurements should include a bacteriological test of the nature of the suspended matter as well as a physical and chemical one.

St. Mary's Hospital Medical School.-The opening of the winter session will take place on Wednesday, Oct. 1st, at 3.30 P.M., when the prizes and awards for 1912-13 will be presented, and an address delivered by Sir John Prescott Hewett, K.C.S.I. The annual dinner of past and present students will be held on Thursday, Oct. 2nd, at the Grand Hall, Princes' Restaurant, at 7 P.M., Dr. Horace Sworder in the chair.

\section{VITAL STATISTICS.}

\section{HFALTH OF ENGLISH TOWNS.}

IN the 96 English and Welsh towns, with populations exceeding 50,000 persons at the last Census and whose aggregate population at the middle of this year is estimated at 17,852,766 persons, 8755 births and 4980 deaths were registered during the week ended Saturday, Sept. 13th. The annual rate of mortality in these towns, which had been $13 \cdot 2,13 \cdot 5$, and $13 \cdot 8$ per 1000 in the three preceding weeks, further rose to $14 \cdot 5$ per 1000 in the week under notice. During the first eleven weeks of the current quarter the mean annual death-rate in these towns averaged $12 \cdot 4$, against 11.5 per 1000 in London during the same period. Among the several towns the death-rates last week ranged from 5.6 in York, 6.0 in Swindon, 6.3 in Darlington, 6.9 in Ealing and in Bournemouth, and 7.0 in Willesden, to 21.7 in Middlesbrough, 22.2 in Dudley and in Sunderland, 22.3 in Liverpool, $23 \cdot 1$ in St. Helens, and 23.5 in Rhondda.

The 4980 deaths from all causes were 247 in excess of the number in the previous week, and included 1242 which were referred to the principal epidemic diseases, against numbers steadily increasing from 281 to 1099 in the ten preceding weeks. Of these 1242 deaths, 1104 resulted from infantile diarrhceal diseases, 38 from whooping-cough, 36 from diphtheria, 28 from scarlet fever, 26 from measles, and 10 from enteric fever, but not one from small-pox. The mean annual death-rate from these diseases last week rose to $3 \cdot 6$, against $2 \cdot 9$ and $3 \cdot 2$ per 1000 in the two preceding weeks. The deaths of infants (under 2 years of age) attributed to diarrhœea and enteritis, which had steadily risen from 149 to 960 in the eight preceding weeks further rose to 1104 last week, and included 229 in London, 78 in Birmingham, 66 in Liverpool, 45 in Manchester, 38 in Hull, 32 in Sheffield, 31 in West Ham, 30 in Rhondda, 27 in Leeds, and 23 in Sunderland. The deaths referred to whooping-cough, which had been 36,50 , and 31 in the three preceding weeks, rose to 38 last week; of this number 7 were registered in London, 6 in Liverpool, 4 in Newcastleon-Tyne, 3 in Birmingham, and 3 in Manchester. The fatal cases of diphtheria, which had been 37,36 , and 45 in the three preceding weeks, declined to 36 last week; 6 deaths occurred in London, 4 in Birmingham, 3 in Manchester, and 2 each in Southampton, Stoke-on-Trent, Rotherham, Middlesbrough, and Cardiff. The deaths attributed to scarlet fever, which had been 31,14 , and 17 in the three preceding weeks, rose to 28 last week, and included 4 in Sheffield 3 in London, 3 in Birmingham, 3 in Sunderland, and 2 each in Plymouth, Nottingham, and Manchester. The deaths referred to measles, which had steadily declined from 77 to 33 in the seven preceding weeks, further fell to 26 last week; 6 deaths were registered in Norwich 5 in Stoke-on-Trent, 3 in London, 2 in Liverpool, and 2 in Manchester. The fatal cases of enteric fever numbered 10 , and were equal to the average in the earlier weeks of the quarter.

The number of scarlet fever patients under treatment in the Metropolitan Asylums and the London Fever Hospitals, which had been 2070, 2115, and 2112 at the end of the three preceding weeks, had risen to 2211 on Saturday last; 346 new cases were admitted during the week, against 239,331 , and 298 in the three preceding weeks. These hospitals also contained on Saturday last 810 cases of diphtheria, 214 of whooping-cough, 118 of measles, and 49 of enteric fever, but not one of small-pox. The 1183 deaths from all causes in London were 98 in excess of the number in the previous week, and were equal to an annual death-rate of 13.7 per 1000 . The deaths referred to diseases of the respiratory system, which had been 128 , 124, and 132 in the three preceding weeks, were again 132 last week, and were 29 above the number in the corresponding week of last year.

Of the 4980 deaths from all causes in the 96 towns, 138 resulted from various forms of violence and 297 were the subject of coroners' inquests. The causes of 28 , or 0.6 per cent., of the total deaths were not certified either by a registered medical practitioner or by a coroner after inquest. All the causes of death were duly certified in Manchester, Sheffield, Leeds, Bristol, West Ham, Bradford, Hull, Newcastle-on-Tyne, and in 73 other smaller towns. Of the 28 